\title{
Scattering polarization of molecular emission lines in the quiet solar chromosphere
}

\author{
M. Faurobert ${ }^{1}$ and J. Arnaud ${ }^{2}$ \\ 1 Département Cassini, UMR 6529, Observatoire de la côte d'Azur, BP 4229, 06304 Nice, France \\ 2 UMR 5572, Observatoire Midi-Pyrénées, 14 avenue Édouard Belin, 31400 Toulouse, France
}

Received 8 November 2001 / Accepted 13 December 2001

\begin{abstract}
We present scattering polarization measurements performed with THEMIS in July 2000 near the south polar limb. The low level of scattered light at THEMIS, which is the only large solar telescope to include a superpolished primary mirror, allows, in good seeing conditions, to observe the emission spectrum of the low chromosphere above the limb. These are, as far as we know, the first high spectral resolution observations of the intensity and the first measurements of the polarization of $\mathrm{C}_{2}$ and $\mathrm{MgH}$ molecular lines in emission above the limb. Molecules are present in a thin layer in the region of the temperature minimum between the photosphere and the chromosphere. We present a very simple model for the formation of the polarized lines and we relate the observed polarization rates to the so-called intrinsic line polarizability coefficients $W_{2}$. Those quantities may be derived from quantum mechanical computations involving the solution of the Schrödinger equation for the molecular potential. Solar observations provide a direct way of checking these heavy computations and contribute to the improvement of our knowledge in molecular physics. Nine $\mathrm{C}_{2}$ molecular transitions and two $\mathrm{MgH}$ transitions are present in our spectral window; we find that for the $\mathrm{C}_{2}$ transitions, the polarizability is between 0.13 and 0.26 and that it takes higher values, namely 0.41 and 0.46 , for the two $\mathrm{MgH}$ transitions.
\end{abstract}

Key words. techniques: polarimetric - techniques: spectroscopic - Sun: atmosphere - Sun: polarization - spectral lines: scattering

\section{Introduction}

Molecular lines are observed in the solar atmosphere, they were first detected in sunspot umbrae but they are also present in quiet regions. Close to the limb, they appear as very weak absorption features in the intensity spectrum. However, their linear polarization dominates clearly in the so-called "second solar spectrum" of linear polarization, with polarization rates on the order of $0.1 \%$ at 5 arcsec inside the solar limb (see Stenflo \& Keller 1996; Stenflo et al. 1997; Gandorfer 2000; Arnaud et al. 2001).

Off-limb spectro-polarimetric observations are quite rare and difficult (see Keller \& Sheeley 1999) because they require a very low level of stray light and good seeing conditions. THEMIS has been especially designed for precise measurements of the four Stokes parameters in the visible solar spectrum (see Arnaud et al. 1998) and its primary mirror is superpolished. Therefore it provides favourable observing conditions for the measurement of the "off-limb" polarization of emission lines in the low

Send offprint requests to: M. Faurobert,

e-mail: faurob@obs-nice.fr chromosphere. We present here such spectro-polarimetric observations which we performed on July 2, 2000, in the spectral range $515.60 \mathrm{~nm}$ to $516.20 \mathrm{~nm}$. The molecular lines appear as emission lines up to one arcsec above the limb, and their linear polarization can be measured; they disappear rapidly at higher altitudes.

In the second part of this paper we give some details about the observing set-up and reduction procedures that we used, and we present the observed intensity and linear polarization spectra recorded at one arcsec above the solar limb.

The emission which is detected is due to scattering of the incident photospheric radiation field by dipoletype electronic transitions of molecules. Assuming that the molecules are present in a thin layer in the low chromosphere, we can show that the polarization of the scattered light depends on the anisotropy of the incident radiation and on the polarizability of the dipole transitions.

The incident radiation field is the photospheric continuum radiation at $516 \mathrm{~nm}$ which is formed in the low photosphere. Its anisotropy can be obtained from a standard radiative transfer modeling under local thermodynamical 
conditions, using semi-empirical models of the temperature and density in the solar photosphere, and well known results for its opacity in the spectral domain of interest. The third part of this paper is devoted to the modeling and interpretation of the observed spectra.

\section{Observations}

The observations were made for slit positions very close to the solar south pole under excellent seeing conditions. The polarization analysis package of THEMIS (cf. Paletou \& Molodij 2001) consists in two identical Fichou achromatic quarter-wave elements, made up of two plates in quartz and in $\mathrm{MgF}_{2}$, followed by a calcite beam splitter. Two beams of orthogonal polarization exit the polarimeter and go through the spectrometer for spectral analysis. They are recorded on one single CCD. High precision spectropolarimetry can be achieved with such a system by applying the beam exchange technique (Semel 1994; Donati et al. 1990). This was performed for the Stokes parameter $Q$. The parameter $Q$ is defined in a standard way, with respect to a system of axis parallel and perpendicular to the solar limb (see next section). In this reference system, for symmetry reasons, both $U$ and $V$ are zero in regions where there is no net magnetic flux. We could check that in the regions where we observed, $U$ and $V$ are indeed below our detection limit, less than one order of magnitude smaller than $Q$. We observed with the solar limb inside the slit field of view and with the slit direction at $45^{\circ}$ of the solar limb. The slit width is 0.5 arcsec and the pixel size is 0.28 arcsec in the direction perpendicular to the limb. The spectral resolution is of $1.6 \mathrm{pm}$ per pixel. The solar limb position is defined as the inflexion point of the limb darkening curve.

The observation procedure that we used, together with the reduction technic that we applied, are described in details in Faurobert et al. (2001). We used an exposure time of $2 \mathrm{~s}$ and added 300 polarization sequences to accumulate enough photons. A polarization sequence consists, for each beam, in two images for $I \pm Q$ (with beam exchange), one for $I \pm U$ and one for $I \pm V$. A polarimetric sensitivity of $0.001 \%$ to $0.002 \%$ can be achieved for long time exposures.

The flat-field images have been built up from scans made while the telescope was moving in a way to have the solar image describing a pseudo-ellipse centered near the solar disc, in a direction chosen to avoid active regions passing across the field of view. The flat-field scans images were stacked for each of the four retarder positions. The four resulting images were destretched to have wavelength variations along the detector rows and spatial variations along the columns. They were then flattened in the usual way, dividing each image by its average row in order to remove the spectral features.

The stray light level is estimated to $2 \%$ of the continum intensity level at one arcsec inside the limb (see Faurobert et al. 2001). For observations performed outside the limb, the light scattered inside the instrument, likely inside the spectrographs, gives rise to a continuum (without any solar lines) background in the intensity spectrum, but it does not affect the polarization spectrum. At one arcsecond above the limb, this continuum account for a little more than $50 \%$ of the total scattered light. The other part comes from sky and telescope scattering and seeing spread of photospheric light. This contribution appears clearly in observations performed higher outside the limb, where no more emission is detected in the molecular lines. At 2.5 arcsec above the limb, we detect a scattered spectrum with absorption lines very similar to the photospheric spectrum very close to the limb (for THEMIS observations at 30 arcsec above the limb, the stray light has also a continuum and a scattered spectrum component, as shown by Paletou et al. 2001).

The intensity spectrum which is presented in Fig. 1 is obtained by subtracting the two components of scattered light, i.e. a pure continuum and a pure scattered spectrum like the one at 2.5 arcsec above the limb. It has been checked that the resulting molecular line emission is quite insensitive to slight solar spectrum shape differences like the ones which exist in the near limb photosphere.

Figure 1 shows the intensity spectrum, normalized by the intensity $I_{\mathrm{c}}$ observed in the continuum at disk center. The molecular lines are clearly seen in emission, together with the FeII, FeI, CoI, Ni I and La II atomic lines. In Fig. 2 we show the opposite of the linear polarization parameter $Q$ normalized by the same quantity. We measured negative values of $Q$ which means that the linear polarization is parallel to the solar limb. We chose to show the Stokes parameter $Q$ instead of the fractional polarization $Q / I$ because, in the chromospheric spectrum, both the intensity $I$ and the linear polarization $Q$ go to zero outside spectral lines. The fractional polarization $Q / I$ should go to zero as well, but small inaccuracies in the measurements of these quantities lead to big and unmeaningful fluctuations of the ratio $Q / I$. Linear polarization is detected in all the molecular lines, with fractional polarization on the order of a few percents (see Table 1). It is also marginally detected in the Fe II, Fe I, Co I and La II lines.

\section{Modeling of line formation}

The emission in the $\mathrm{C}_{2}$ and $\mathrm{MgH}$ lines is detected in the low chromosphere only, it decreases very fast at altitudes higher than $800 \mathrm{~km}$ above the solar limb. This is a direct evidence that these molecules are present only in a thin layer in the temperature minimum region between the photosphere and the chromosphere. Other molecules, such as CO, are observed in the low solar chromosphere. Their existence implies that low temperature regions do exist in this area. One possible cooling mechanism is the overshooting of granular material that expands near the height of the temperature minimum (see Solanki et al. 1996). The width of the emission lines shown in Fig. 1 is in very good agreement with this scenario. We measure a typical line width at half maximum of $96 \mathrm{~m} \AA \pm 5 \mathrm{~m} \AA$, which seems to be quite similar for the molecular and the atomic lines. 


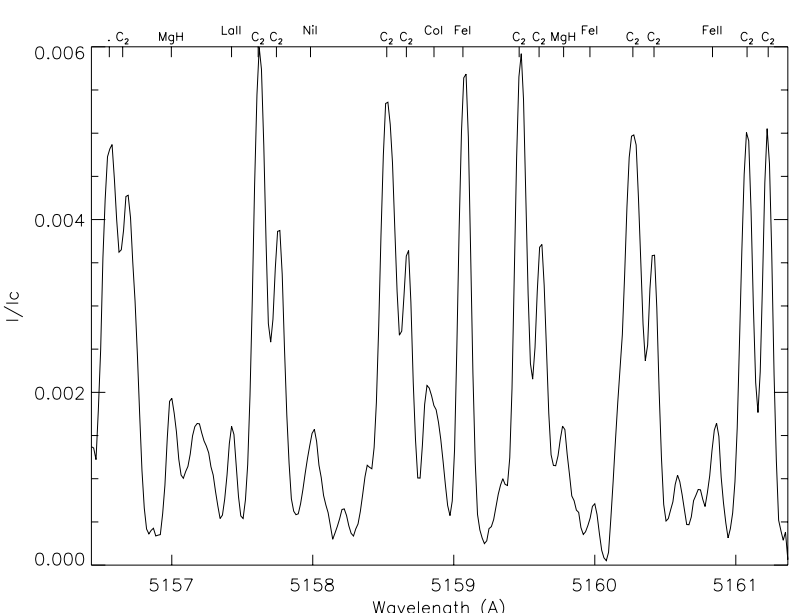

Fig. 1. $I / I_{\mathrm{c}}$ recorded at one arcsec above the solar limb, $I_{\mathrm{c}}$ is the intensity of the continuum at disk center. Molecular lines of $\mathrm{C}_{2}$ and $\mathrm{MgH}$ appear as emission lines, together with atomic lines of FeII, FeI, CoI, Ni I and LaII.

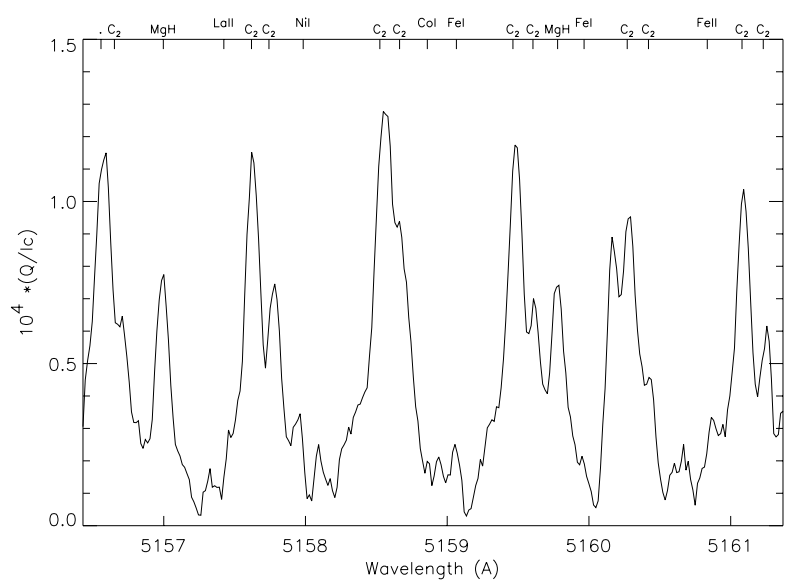

Fig. 2. $|Q| / I_{\mathrm{c}}$ recorded at one arcsec above the solar limb, $I_{\mathrm{c}}$ is the intensity of the continuum at disk center. We observe the scattering polarization of $\mathrm{C}_{2}$ and $\mathrm{MgH}$ lines. The polarization of the atomic lines of Fe II, Fe I, Co I and La II is also marginaly detected.

Assuming that line broadening is mainly due to macroturbulent velocity fields, we derive the mean quadratic value of the horizontal component of the velocity, which is equal to $3.1 \mathrm{~km} \mathrm{~s}^{-1} \pm 0.2 \mathrm{~km} \mathrm{~s}^{-1}$. This value is consistent with the idea that molecules are formed in the overshooting granules which expand near the height of the temperature minimum. Large horizontal velocities are reminiscent of the almost adiabatically expanding cool gas overlying granules in the upper reaches of granular simulations (see Stein \& Nordlund 1989).

Up to now very few theoretical studies have been devoted to the formation of polarized molecular lines. Mohan Rao \& Rangarajan (1999) proposed an interpretation of the linear polarization observed by Stenflo \& Keller (1997) in $\mathrm{MgH}$ absorption lines at $516.5933 \mathrm{~nm}$ and $516.8147 \mathrm{~nm}$, close to the solar limb. They assumed that $W_{2}=0.4$ for these lines. This value is obtained when one simply generalizes to molecular electronic transitions the expression which is valid for atomic lines.

Let us notice that one of the assumptions of this quoted work is that the molecular lines are formed in the lower solar atmosphere where near LTE conditions prevail, this is in contradiction with our observations.

In our model, the thin molecular layer is illuminated by the incident continuous radiation emitted by the photosphere. Due to limb-darkening, the photospheric radiation field is anisotropic. Inside the slab, the incident radiation is scattered by molecular dipole-type transitions. This scattering gives rise to the emission of polarized radiation that we observe. In the spectral domain where we observed, the molecular lines of $\mathrm{C}_{2}$ and $\mathrm{MgH}$ are due to electronic transitions $A^{3} \Pi_{g}-X^{3} \Pi_{u}$ and $A^{2} \Pi_{3 / 2,1 / 2}-X^{2} \Sigma$, respectively, in the vibration band $(0,0)$. The $\mathrm{C}_{2}$ lines belong to the $\mathrm{P}$ branch. In our observations they appear as doublets; the "blue" line of the doublets correspond to P1 and P2 components, which are blended, and the "red" component belongs to the P3 branch. The identification and rotational quantum numbers for all the molecular lines of our spectral domain are taken from Pierce (1968) and given in Table 1.

We shall assume that the molecular layer is optically thin, so that the photospheric radiation undergoes at most one scattering. This is justified by the observations performed on the disk, close to the solar limb, where the molecular absorption lines are very weak (Stenflo et al. 1997). Furthermore, as the density of neutral hydrogen in the chromosphere is very low, we neglect elastic collisions which could destroy the polarization. Molecular lines in the visible domain are almost not sensitive to the effect of magnetic fields because the Landé factors of the transitions are very small (Stenflo, personnal communication), so we shall not consider any depolarizing mechanism due to the Hanle effect of weak and unresolved magnetic fields which could be present in the low chromosphere.

The incident radiation field is symmetrical with respect to the radial axis and varies with respect to the angle $\theta$ between its propagation direction and the local solar radius. It does not dependent on frequency on the spectral interval of interest. As we observe off-limb, the line of sight is perpendicular to the radial direction. The continuum photospheric spectrum is very weakly polarized in this spectral domain (see Leroy 1972), so that we can safely neglect the polarization of the incident radiation. Its specific intensity is denoted by $I_{\text {inc }}(\mu)$ where $\mu$ is the cosine of $\theta$.

The emissivity is due both to scattering of the incident radiation and to thermal emission processes. Thermal processes do not give rise to polarization, so here we consider only the emissivity due to molecular scattering. The scattering phase matrix is denoted by $\hat{P}\left(\mu, \mu^{\prime}\right)$, where $\mu$ and $\mu^{\prime}$ refer respectively to the propagation directions of the reemitted and of the incident radiation fields. The Stokes 
parameters of the scattered radiation are related to those of the incident radiation by

$\boldsymbol{I}_{\mathrm{sc}}(\mu)=\hat{P}\left(\mu, \mu^{\prime}\right) \boldsymbol{I}_{\mathrm{inc}}\left(\mu^{\prime}\right)$,

where $\boldsymbol{I}_{\mathrm{sc}}(\mu)=\left(I_{\mathrm{sc}}(\mu), Q_{\mathrm{sc}}(\mu)\right)^{\dagger}$ and $\boldsymbol{I}_{\text {inc }}\left(\mu^{\prime}\right)=$ $\left(I_{\text {inc }}\left(\mu^{\prime}\right), 0\right)^{\dagger}$. We define here the Stokes parameters $I$ and $Q$, as in Chandrasekhar (1960); $I$ is the specific intensity of the radiation field, and $Q=I_{1}-I_{\mathrm{r}}$, where $I_{\mathrm{l}}$ and $I_{\mathrm{r}}$ denote the components which are respectively perpendicular and parallel to the limb. According to this definition, negative values of $Q$ correspond to a linear polarization parallel to the limb.

The corresponding source function, denoted by $\boldsymbol{S}_{\mathrm{sc}}(\mu)$ is given by

$\boldsymbol{S}_{\mathrm{sc}}(\mu)=\frac{k_{\mathrm{sc}}}{k_{\mathrm{sc}}+k_{\mathrm{a}}} \int_{-1}^{1} \hat{P}\left(\mu, \mu^{\prime}\right) \boldsymbol{I}_{\mathrm{inc}}\left(\mu^{\prime}\right) \frac{\mathrm{d} \mu^{\prime}}{2}$.

In $(2), k_{\mathrm{sc}}$ is the molecular scattering coefficient and $k_{\mathrm{a}}$ is the absorption coefficient of the low chromosphere at the same wavelength.

The general form of the scattering phase matrix for dipolar electric transitions is

$\hat{P}\left(\mu, \mu^{\prime}\right)=\hat{P}_{\text {is }}+\frac{3}{8} W_{2} \hat{P}_{0}\left(\mu, \mu^{\prime}\right)$,

where $\hat{P}_{\text {is }}$ is the isotropic phase matrix (only the first element is different from zero and equal to 1 ), and $\hat{P}_{2}^{(0)}$ is given by

$\hat{P}_{0}\left(\mu, \mu^{\prime}\right)=\left(\begin{array}{cc}\left(\frac{1}{3}-\mu^{2}\right)\left(1-3 \mu^{\prime 2}\right) & \left(1-3 \mu^{2}\right)\left(1-\mu^{\prime 2}\right) \\ \left(1-\mu^{2}\right)\left(1-3 \mu^{\prime 2}\right) & 3\left(1-\mu^{2}\right)\left(1-\mu^{\prime 2}\right)\end{array}\right)$

The coefficient $W_{2}$ is a constant, called the intrinsic polarizability, which depends on the quantuum numbers of the lower and upper levels of the molecular transition. In the case of pure Rayleigh scattering $W_{2}=1$, in the general case it is smaller than unity.

The stokes parameters of the radiation emitted by the molecular region are obtained through the formal solution of the transfer equation, i.e.

$\boldsymbol{I}_{\mathrm{sc}}(\mu)=\int_{0}^{T} \boldsymbol{S}_{\mathrm{sc}}(\mu) \mathrm{e}^{-\tau / \mu} \frac{\mathrm{d} \tau}{\mu}$

where $T$ is the optical thickness of the region. For off-limb observations, where $\mu=0$, we have

$\boldsymbol{I}_{\mathrm{sc}}(\mu)=\boldsymbol{S}_{\mathrm{sc}}(\mu)$

Let us introduce the integrals

$I_{0}=\int_{-1}^{1} I_{\text {inc }}\left(\mu^{\prime}\right) \mathrm{d} \mu^{\prime} / 2$

and

$I_{1}=\int_{-1}^{1}\left(1-3 \mu^{\prime 2}\right) I_{\text {inc }}\left(\mu^{\prime}\right) \mathrm{d} \mu^{\prime} / 2$.

We notice that, as the incident radiation comes from the photosphere, it is zero for negative values of $\mu^{\prime}$. According
Table 1. Linear polarization rates observed in the molecular emission lines and corresponding polarizability coefficients. The fourth column gives the branching ratios $r=k_{\mathrm{sc}} /\left(k_{\mathrm{sc}}+k_{\mathrm{a}}\right)$ derived from the intensity observed in the lines.

\begin{tabular}{ccccc}
\hline Molecular line & Branch $(J)$ & $|Q| / I(\%)$ & $W_{2}$ & $r(\%)$ \\
\hline$(\mathrm{MgH}) 515.70 \mathrm{~nm}$ & $\mathrm{Q}(17)$ & 3.96 & 0.41 & 0.74 \\
$\left(\mathrm{C}_{2}\right) 515.76 \mathrm{~nm}$ & $\mathrm{P} 1(30)+\mathrm{P} 2(29)$ & 1.90 & 0.20 & 2.3 \\
$\left(\mathrm{C}_{2}\right) 515.77 \mathrm{~nm}$ & $\mathrm{P} 3(28)$ & 1.91 & 0.20 & 1.5 \\
$\left(\mathrm{C}_{2}\right) 515.85 \mathrm{~nm}$ & $\mathrm{P} 1(29)+\mathrm{P} 2(28)$ & 2.40 & 0.25 & 2.1 \\
$\left(\mathrm{C}_{2}\right) 515.87 \mathrm{~nm}$ & $\mathrm{P} 3(27)$ & 2.55 & 0.26 & 1.4 \\
$\left(\mathrm{C}_{2}\right) 515.95 \mathrm{~nm}$ & $\mathrm{P} 1(28)+\mathrm{P} 2(27)$ & 1.99 & 0.21 & 2.3 \\
$\left(\mathrm{C}_{2}\right) 515.96 \mathrm{~nm}$ & $\mathrm{P} 3(26)$ & 1.87 & 0.19 & 1.4 \\
$(\mathrm{MgH}) 515.98 \mathrm{~nm}$ & $\mathrm{Q}(16)$ & 4.54 & 0.46 & 0.62 \\
$\left(\mathrm{C}_{2}\right) 516.02 \mathrm{~nm}$ & $\mathrm{P} 1(27)+\mathrm{P} 3(1)$ & & & \\
$\left(\mathrm{C}_{2}\right) 516.03 \mathrm{~nm}$ & $\mathrm{P} 2(26)$ & 1.90 & 0.20 & 1.9 \\
$\left(\mathrm{C}_{2}\right) 516.04 \mathrm{~nm}$ & $\mathrm{P} 3(25)$ & 1.26 & 0.13 & 1.4 \\
$\left(\mathrm{C}_{2}\right) 516.10 \mathrm{~nm}$ & $\mathrm{P} 1(26)+\mathrm{P} 2(25)$ & 2.06 & 0.21 & 1.9 \\
\hline \hline
\end{tabular}

to (6), the polarization rate which is observed off-limb, in the molecular lines is given by

$Q / I(\mu=0)=3 \frac{W_{2}}{8} \frac{I_{1}}{I_{0}+\frac{W_{2}}{8} I_{1}}$,

it depends on $W_{2}$ and on the values of the integrals $I_{0}$ and $I_{1}$.

We calculated the photospheric continuous radiation at $516 \mathrm{~nm}$ from the solution of the radiative transfer equation in local thermodynamical equilibrium and for a standard model of the solar quiet photosphere (Fontenla et al. 1993). From these calculations we derive the center-tolimb variation of $I_{\text {inc }}$ and we obtain the values of $I_{0}$ and $I_{1}$. This gives

$I_{0}=0.35 I_{\text {inc }}(\mu=1), \quad I_{1}=-0.09 I_{\text {inc }}(\mu=1)$,

where $I_{\text {inc }}(\mu=1)$ is the photospheric radiation at disk center. The negative sign of $I_{1}$ is a consequence of the limb-darkening of the incident photospheric radiation and it explains that we observe negative values for the Stokes parameter $Q$ in the molecular lines.

\section{Discussion of the results}

From the intensity observed in the lines we derived the value of the branching ratio of molecular scattering in the layer, $k_{\mathrm{sc}} /\left(k_{\mathrm{sc}}+k_{\mathrm{a}}\right)$. It takes values of $1.4 \%$ to $1.5 \%$ for all the unblended P3 lines of $\mathrm{C}_{2}$ and of $2.1 \%$ to $2.3 \%$ for the blended (P1-P2) ones. The $\mathrm{C}_{2}$ line at $516.02 \mathrm{~nm}$ has a somewhat different behaviour. It is formed of two blended components $\mathrm{P} 1(J=27)$ and $\mathrm{P} 3(J=1)$, and it is very close to the P2 $(J=26)$ line at $516.03 \mathrm{~nm}$. As the emission lines are quite broad, they appear as one single peak in the intensity spectrum. But the polarization peak spectrum 
shows indeed two peaks at $516.02 \mathrm{~nm}$ and $516.03 \mathrm{~nm}$ (see Fig. 2).

The branching ratio of molecular scattering is significantly smaller for the $\mathrm{MgH}$ lines, with values of $0.74 \%$ and $0.62 \%$. The reason for this difference is probably that $\mathrm{MgH}$ molecules are less abondant than $\mathrm{C}_{2}$ in the low chromosphere.

Table 1 also gives the absolute values of the polarization rates observed at 1 . arcsec above the limb in the $\mathrm{C}_{2}$ and $\mathrm{MgH}$ lines and the values of $W_{2}$ deduced from (9). We did not consider the two lines which are very close to the blue and red limits of our spectral window because they may be affected by vignetting effects. For most of the $\mathrm{C}_{2}$ lines in our spectral window we find that the polarizabilities of the blended (P1 and P2) and unblended (P3) components which appear in doublets have similar values, between 0.19 and 0.20 . However two doublets, at (515.85-515.87) nm and (516.03-516.04) nm respectively, show a different behaviour. For the first one, we obtain slightly higher values $(0.25$ and 0.26$)$, but we can see on Fig. 2 that the two polarization peaks are barely resolved and that they might be affected by the presence of blended polarizing lines both on the blue and the red sides. This could lead to an overestimation of the intrinsic polarizability of these $\mathrm{C}_{2}$ lines. As far as the second doublet is concerned, the value of $W_{2}$ that we derive for the P3 line at $516.04 \mathrm{~nm}$ is signifcantly lower (0.13) than the "nominal" value obtained for the other P3 lines of our spectral window (0.19). But the polarization peak of the P3 line at $516.04 \mathrm{~nm}$ is only marginally detected (see Fig. 2). The value that we derive for $W_{2}$, is thus more questionable. As we cannot measure the intensity of the $\mathrm{P} 1+\mathrm{P} 2$ component at $516.02 \mathrm{~nm}$, we cannot estimate the corresponding value for $W_{2}$ (see the discussion above).

We find that the intrinsic polarizability of the $\mathrm{MgH}$ lines is about twice larger than that of the $\mathrm{C}_{2}$ lines. It is not very far from the value 0.4 which was used by Mohan Rao \& Rangarajan (1999), by analogy with atomic lines. We stress that this analogy would not give correct results for the $\mathrm{C}_{2}$ lines. Theoretical calculations of the polarizability of molecular lines are needed to compare with our empirical determination.
Acknowledgements. We thank J. O. Stenflo for stimulating discussions about molecular scattering and the THEMIS technical staff and the support astronomer F. Paletou for their help. THEMIS is operated on the Island of Tenerife by CNRSCNR in the Spanish Observatorio del Teide of the Instituto de Astrofísica de Canarias.

\section{References}

Arnaud, J., Mein, P., \& Rayrole, J. 1998, in a Crossroads For European Solar and Heliographic Physics, Tenerife, March 1998, ed. ESA SSPG and IAC, ESA SP-417, 213

Arnaud, J., Faurobert, M., Vigneau, J., \& Paletou, F. 2001, in Advanced Solar Polarimetry - Theory, Observation and Instrumentation, ed. M. Sigwarth, ASP Conf. Ser., 236, 151

Chandrasekhar, S. 1960, Radiative Transfer (Dover, New York)

Donati, J.-F., Semel, M., Rees, D. E., Taylor, K., \& Robinson, R. D. 1990, A\&A, 232, L1

Faurobert, M., Arnaud, J., Vigneau, J., \& Frisch, H. 2001, A\&A, 378, 627

Fontenla, J. M., Avrett, E. H, \& Loeser, R. 1993, ApJ, 406, 319

Gandorfer, A. 2000, The Second Solar Spectrum, a high spectral resolution polarimetric survey of scattering polarization at the solar limb in graphical representation, Hochschulverlag AG and der ETH, Zürich, VdF. ISBN No. 3, 7281, 27647

Keller, C. U., \& Sheeley Jr., N. R. 1999, in Solar Polarization, ed. K. N. Nagendra, \& J. O. Stenflo (Kluwer), 17

Leroy, J. L. 1972, A\&A, 19, 287

Mohan Rao, D., \& Rangarajan, K. E. 1999, ApJ, 524, L139

Paletou, F., \& Molodij, G. 2001, in Advanced Solar Polarimetry - Theory, Observation and Instrumentation, ed. M. Sigwarth, ASP Conf. Ser., 236, 9

Paletou, F., Lopez-Ariste, A., Bommier, V., \& Semel, M. 2001, A\&A, 375, L39

Pierce, A. 1968, ApJS, 17, 1

Semel, M. 1994, in Solar Surface Magnetism, ed. R. J. Rutten, \& C. J. Schrijver (Kluwer)

Solanki, S. K., Livingston, W., Muglach, K., \& Wallace, L. 1996, A\&A, 315, 303

Stein, R., \& Nordlund, A. 1989, ApJ, 342, L95

Stenflo, J. O., \& Keller, C. 1996, Nature, 382, 588

Stenflo, J. O., Bianda, M., Keller, C. U., \& Solanki, S. K. 1997, A\&A, 322, 994 